\title{
Remote ischemic preconditioning to reduce contrast-induced nephropathy: study protocol for a randomized controlled trial
}

\author{
Thomas B Sterenborg ${ }^{1 \dagger}$, Theo P Menting ${ }^{1 \dagger}$, Yvonne de Waal ${ }^{2}$, Rogier Donders ${ }^{3}$, Kimberley E Wever ${ }^{1}$,
} M Susan Lemson ${ }^{5}$, Daan JA van der Vliet', Jack F Wetzels ${ }^{2}$, Leo J SchultzeKool ${ }^{4}$ and Michiel C Warlé ${ }^{*}$

\begin{abstract}
Background: Despite the increasing use of pre- and posthydration protocols and low-osmolar instead of high-osmolar iodine-containing contrast media, the incidence of contrast-induced nephropathy (CIN) is still significant. There is evidence that contrast media cause ischemia-reperfusion injury of the medulla. Remote ischemic preconditioning (RIPC) is a non-invasive, safe, and low-cost method to reduce ischemia-reperfusion injury.

Methods: The RIPCIN study is a multicenter, single-blinded, randomized controlled trial in which 76 patients at risk of CIN will receive standard hydration combined with RIPC or hydration with sham preconditioning. RIPC will be applied by four cycles of 5 min ischemia and 5 min reperfusion of the forearm by inflating a blood pressure cuff at $50 \mathrm{mmHg}$ above the actual systolic pressure. The primary outcome measure will be the change in serum creatinine from baseline to 48 to $72 \mathrm{~h}$ after contrast administration.
\end{abstract}

Discussion: A recent pilot study reported that RIPC reduced the incidence of CIN after coronary angioplasty. The unusual high incidence of CIN in this study is of concern and limits its generalizability. Therefore, we propose a randomized controlled trial to study whether RIPC reduces contrast-induced kidney injury in patients at risk for CIN according to the Dutch guidelines.

Trial registration: Current Controlled Trials ISRCTN76496973

Keywords: Contrast-induced nephropathy, Remote ischemic preconditioning, Acute kidney injury, Pre- and posthydration, Randomized controlled trial

\section{Background}

Iodine-containing contrast media are often used for diagnostic and therapeutic procedures and their use is the leading cause of hospital-acquired acute kidney injury [1]. Prospective studies demonstrate that contrast media are responsible for approximately $15 \%$ of acute kidney injury cases [2,3]. Despite the increasing use of pre- and posthydration protocols and low-osmolar instead of high-osmolar iodine-containing contrast media, the incidence of contrast-induced acute kidney injury is still significant $[4,5]$. This so called contrast-induced nephropathy

\footnotetext{
* Correspondence: m.warle@chir.umcn.nl

${ }^{\dagger}$ Equal contributors

'Department of Surgery, Division of Vascular- and Transplant Surgery, Radboud University Nijmegen Medical Centre, Geert Grooteplein-Zuid 10, Nijmegen, GA 6525, the Netherlands

Full list of author information is available at the end of the article
}

(CIN) is defined as an absolute rise of $\geq 0.5 \mathrm{mg} / \mathrm{dL}$ and/or a relative increase of $\geq 25 \%$ in serum creatinine compared to baseline within 48 to $72 \mathrm{~h}$ after contrast administration without an alternative cause of kidney injury [6]. CIN is strongly associated with morbidity and mortality $[7,8]$. In patients with CIN, $8 \%$ need dialysis treatment and between $22 \%$ and $34 \%$ die during the index hospitalization [3,9-11]. In accordance with international guidelines, all patients who receive iodine-containing contrast are screened for risk factors of CIN, including measures of renal function (estimated glomerular filtration rate, based upon the MDRD formula) [12-15]. High-risk patients receive preand posthydration by saline solution infusion for 4 to $12 \mathrm{~h}$. Furthermore, 48 to $72 \mathrm{~h}$ after contrast administration, serum creatinine should be measured [16]. Despite the identification of high-risk patients and the use of hydration 
protocols, the incidence of CIN still varies between $2 \%$ and $13 \%$ [17-20]. The exact mechanism underlying CIN remains to be elucidated. There is evidence to suggest that contrast media have direct toxic effects on the tubular cells resulting in altered mitochondrial function and apoptosis [21]. Moreover, ischemia-reperfusion injury of the medulla has been shown to play an important role [22]. The outer part of the medulla has an area with a high oxygen demand and is located at a distance from the vasa recta which supplies the medulla of blood. Contrast-induced vasoconstriction of the vasa recta induces ischemia-reperfusion injury of the medulla which contributes significantly to the pathophysiology of CIN. Remote ischemic preconditioning (RIPC) is a short and harmless discontinuation of blood supply to particular organs or tissue, followed by reperfusion [23,24]. A preconditioning stimulus is applied before the onset of prolonged ischemia. In animal models it has been found to reduce ischemia-reperfusion injury of the kidney [25]. Although the precise mechanism of RIPC remains unknown, two major pathways may play a pivotal role: the humoral and neurogenic pathways. Both are thought to induce various kinase cascades and eventually prevent opening of the mitochondrial permeability transition pore in the target organ, thereby reducing cell death [26]. A retrospective cohort study by Whittaker et al. indicated that multiple balloon inflations during coronary angioplasty (as a remote stimulus) might reduce CIN [27]. Furthermore, a recent pilot study by Er et al. showed that RIPC reduced CIN in high-risk patients undergoing elective coronary angiography [28]. However, there was an unusually high incidence of CIN (40\%) in the control group. The question arises whether protection by RIPC, as an adjunct to standard preventive measures (that is, hydration and discontinuation of nephrotoxic drugs), also holds for patients with a lower risk of CIN. As generalizability of the results by Er et al. is confined to a selected group of patients with an unusual high risk of CIN, we propose a randomized controlled trial to study whether RIPC reduces contrast-induced kidney injury in patients at risk of CIN according to the Dutch guideline [14].

\section{Methods/Design}

A multicenter, single-blinded, randomized controlled trial will be performed at the Radboud University Nijmegen Medical Centre and Slingeland Hospital Doetinchem. Inclusion will be performed by the physician researcher after written informed consent.

\section{Study population}

A total of 76 patients will be randomized. Sealed envelopes are used to randomly assign consecutive patients in a 1:1 ratio to receive either sham preconditioning or RIPC (Figure 1). The study population consists of patients at risk of CIN according to criteria adopted from the Dutch guidelines: (1) eGFR $<45 \mathrm{~mL} / \mathrm{min} / 1.73 \mathrm{~m}^{2}$; (2) eGFR $<60 \mathrm{~mL} / \mathrm{min} / 1.73 \mathrm{~m}^{2}$; (3) eGFR $<60 \mathrm{~mL} / \mathrm{min} /$ $1.73 \mathrm{~m}^{2}$ and two additional risk factors (that is, peripheral vascular disease, heart failure, $>75$ years of age, anemia, dehydration, use of diuretics and/or NSAIDs). Patients undergoing contrast procedures for diagnostic and/or treatment purposes are eligible. As patients receiving less than $100 \mathrm{~mL}$ of iodinated contrast media may not have an increased risk of contrast-induced kidney injury, an expected use of at least $100 \mathrm{~mL}$ was used as inclusion criterion [3,29].

\section{Inclusion criteria}

1) Patients undergoing an interventional or diagnostic radiological procedure in which they receive an expected >100 mL intravascular contrast including:

- Thoracic and/or abdominal endovascular aortic repair

- Endovascular aortic repair

- Digital subtraction angiography

- Percutaneous transluminal angioplasty

- Percutaneous intentional extraluminal revascularization

- Carotic artery stenting

- Percutaneous coiling/embolization procedures

- Computed tomography

2) Patients who comply with the risk criteria for CIN according to the Dutch guidelines [14]

- Peripheral vascular disease, heart failure, $>75$ years, anemia ( $\mathrm{Ht}<0.39$ men and $<0.36$ women, dehydration, diuretics and/or NSAID use)

3) Written informed consent.

\section{Exclusion criteria}

- Age $<18$ years

- Hemodialysis or peritoneal dialysis

- Simultaneous participation in another interventional study

- Percutaneous coiling/embolization procedures of the kidney

- Impossibility to perform RIPC, due to pathology of both arms (for example, dystrophy, recent trauma, chronic wounds)

\section{Study protocol}

All participating patients will receive the standard hydration schedule consisting of an infusion with saline $0.9 \%$ solution 3 to $4 \mathrm{~mL} / \mathrm{kg} / \mathrm{h}$ for $4 \mathrm{~h}$ prior to and $4 \mathrm{~h}$ after contrast administration. In patients with congestive heart failure or MDRD $<30 \mathrm{~mL} / \mathrm{min} / 1.73 \mathrm{~m}^{2}$ a long schedule is used with an infusion of saline $0.9 \%$ solution $1 \mathrm{~mL} / \mathrm{kg} / \mathrm{h}$ for $12 \mathrm{~h}$ prior to and $12 \mathrm{~h}$ after the contrast 


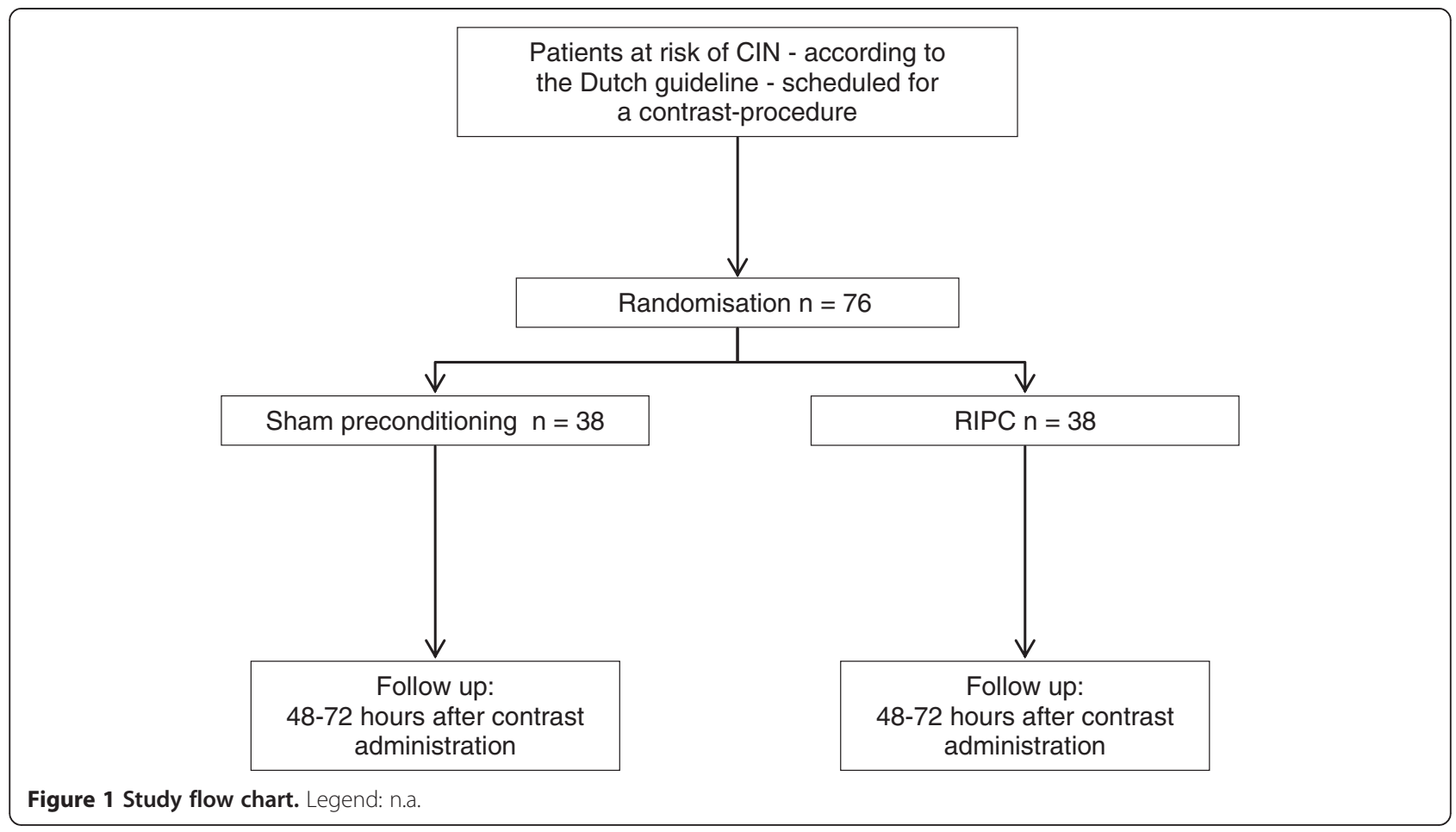

administration. Nephrotoxic drugs (for example, metformin and diuretics) are discontinued at least $24 \mathrm{~h}$ before and after contrast administration [14]. Patients in the experimental group of the study will receive RIPC by four cycles of ischemia and reperfusion of the forearm by inflating a blood pressure cuff around the upper arm at $50 \mathrm{mmHg}$ above the actual systolic pressure during $5 \mathrm{~min}$ followed by $5 \mathrm{~min}$ of reperfusion. In the control group, patients receive sham preconditioning by inflating the blood pressure cuff to $10 \mathrm{mmHg}$ below the actual diastolic pressure during $5 \mathrm{~min}$ followed by $5 \mathrm{~min}$ of reperfusion (four cycles). The time between the last inflation cycle and the start of the intervention is planned within $45 \mathrm{~min}$. In the interest of blinding, the investigator ensures that the inflation pressure is not visible for both the patient and the (interventional) radiologist. All patients receive Xenetrix 300 (0.6 to 0.85 Osmol/kg $\mathrm{H}_{2} \mathrm{O}$ ), a low osmolar, non-ionic, and hydrophilic contrast medium [30,31]. Patients will complete a questionnaire to obtain all relevant baseline characteristics such as age, weight, previous contrast procedures, diabetes, vascular-related diseases, and (discontinuation of) medication. Chart review will be performed to complement and double check this information. Blood and urine samples are taken at baseline and 4 to $6 \mathrm{~h}$ after contrast administration. A final blood sample is taken 48 to $72 \mathrm{~h}$ after contrast administration. According to the Dutch guidelines, monitoring of renal function in highrisk patients is recommended within 48 to $72 \mathrm{~h}$ after contrast administration. All samples will be number coded before analysis to ensure blinding of the independent investigator performing the analyses.

\section{Primary endpoint}

The primary endpoint is change in serum creatinine form baseline to serum creatinine within 48 to $72 \mathrm{~h}$ after contrast administration.

\section{Secondary endpoint}

The secondary endpoints are the incidence of CIN (defined as an absolute rise of $\geq 0.5 \mathrm{mg} / \mathrm{dL}$ or a relative increase of $\geq 25 \%$ in serum creatinine over baseline within 48 to $72 \mathrm{~h}$ after contrast administration), rehospitalization, hemodialysis, and mortality within 6 weeks after contrast administration.

\section{Ethics, informed consent}

An independent ethics committee, the Central Committee on Research involving Human Subjects, ArnhemNijmegen, approved the protocol. Oral and written informed consent from the patient will be obtained prior to inclusion.

\section{Adverse events}

Although RIPC by repeated insufflations of a blood pressure cuff around the upper arm is considered safe, serious adverse events possibly related to the application of RIPC will be reported to the ethical committee. Mild adverse events are: transient discomfort due to 
compression and/or ischemia and the formation of ecchymosis (upper arm) or petechia (lower arm).

\section{Power analysis}

In this randomized study, the change of serum creatinine from baseline to 48 to $72 \mathrm{~h}$ after contrast administration will be compared between the experimental and control group. Using serum creatinine change as continuous response variable increases the power of the study. In a previous retrospective cohort study at our center including 2,169 patients at risk for contrast-induced nephropathy, serum creatinine values decreased from $120 \mu \mathrm{mol} / \mathrm{L}$ at baseline to $118 \mu \mathrm{mol} / \mathrm{L}$ at 48 to $72 \mathrm{~h}$ after contrast administration due to adequate hydration protocols [17]. This mean change in serum creatinine $(-2 \mu \mathrm{mol} / \mathrm{L})$ was normally distributed with a standard deviation of $23 \mu \mathrm{mol} / \mathrm{L}$. Based on existing evidence we assume that RIPC with hydration may provide a further decrease in mean serum creatinine from baseline to 48 to $72 \mathrm{~h}$ of approximately $14 \mu \mathrm{mol} / \mathrm{L}$ as compared to hydration only. This corresponds with approximately $60 \%$ of the effect that was found by Er et al. [28]. If the true difference in the experimental and control means is $14 \mu \mathrm{mol} / \mathrm{L}$, we will need to study 34 experimental and 34 controls to be able to reject the null hypothesis with a power of 0.80 and an alpha of 0.05 calculated with a one-sided independent t-test. Based on existing animal [25] and human studies [32,33] investigating the influence of RIPC on renal ischemiareperfusion injury, we assume that RIPC does not negatively affect renal function. Therefore, one-sided testing would be appropriate for this study. Expected lost to follow-up (for example, blood sampling not realized between 48 to $72 \mathrm{~h}$ ) is approximately $5 \%$. For this reason 38 patients will be included in both the experimental and control arm.

\section{Statistical analysis}

The analysis will be performed on the basis of intentionto-treat principles. Student's t-test will be used to compare normally distributed variables, and Mann-Whitney $\mathrm{U}$ test will be used to compare not-normally distributed continuous data. Categorical variables will be compared with the chi-square test. If univariable analysis reveals a significant difference in baseline characteristics, then a multivariable linear regression analysis will be used to assess its impact on the primary outcome measure (that is, change in serum creatinine between baseline and 48 to $72 \mathrm{~h}$ after contrast administration). A subgroup analysis will be performed to assess whether the impact of RIPC on the primary outcome measure is affected by the Mehran risk score. For this analysis patients will be divided into three equal groups (that is, tertiles) according to their Mehran risk score. Statistical analyses will be performed with SPSS 20.0. A probability value of $<0.05$ is considered to indicate statistical significance and $95 \%$ confidence intervals will be calculated.

The RIPCIN study is registered at: http://www.controlled-trials.com/ISRCTN76496973.

\section{Discussion}

In this study, we hypothesize that RIPC reduces the occurrence of CIN in patients at risk of acute kidney injury due to the use of contrast media. A recent randomized pilot study suggested that RIPC reduced contrastinduced kidney injury, however this study was performed in patients with an unusual high risk of CIN. A comment on this study by Mehta Oza et al. clarified that based on the reported Mehran risk score the incidence of CIN should lie between $26 \%$ and $30 \%$ instead of $40 \%$ as reported by Er et al. [34]. The authors stated that this high incidence of CIN could be attributed to a high prevalence of heart failure and diabetes mellitus in their cohort. However, if standard measures to prevent CIN, that is, hydration with saline and discontinuation of nephrotoxic drugs, were not carried out appropriately, then the incidence of CIN would also be increased. As compliance to standard preventive measures against CIN was not described by Er et al. their results do not fully justify the conclusion that RIPC, as an adjunct to standard preventive measures, effectively reduces CIN. Another important issue to address is the fact that the incidence of CIN varies with the criteria used [35]. Er et al. defined CIN as an absolute or relative increase in serum creatinine, whereas some evidence exists that both an absolute and a relative increase in serum creatinine more accurately predicts adverse events after coronary angioplasty. To overcome the flaws related to the use of different definitions of CIN, we will use the change in serum creatinine from baseline to 48 to $72 \mathrm{~h}$ after contrast administration as that primary endpoint in the proposed trial. As serum creatinine levels generally peak between 48 and $72 \mathrm{~h}$ after contrast administration, it would be ideal to measure serum creatinine at both 48 and $72 \mathrm{~h}$. However, this would not be in line with Dutch and international guidelines which recommend checking renal function once between 48 and $72 \mathrm{~h}$ after contrast administration. In practice most patients are discharged within $24 \mathrm{~h}$ after contrast administration and for many it is already difficult to realize one blood sample between 48 and $72 \mathrm{~h}$ after contrast administration. In our view, it is appropriate for proof-of-concept studies investigating new strategies to reduce contrast-induced kidney injury to use the change in serum creatinine from baseline to 48 to $72 \mathrm{~h}$ as the primary endpoint. Once the efficacy of a new strategy against contrast-induced kidney injury has been confirmed, much larger clinical trials should be conducted with adverse effects after the use of contrast-media (for example, dialysis and/or death) as the primary endpoint. 


\section{Trial status}

The trial is ongoing. Currently 61 patients have been included.

\section{Abbreviations}

CIN: Contrast-induced nephropathy; MDRD: Mean glomerular filtration rate; RIPC: Remote ischemic preconditioning.

\section{Competing interests}

The authors of this manuscript have no competing interests to disclose.

\section{Authors' contributions}

TS contributed to data collection and analysis, manuscript writing, and final approval of the manuscript. TM and YW contributed to data collection, critical revision, and final approval of the manuscript. RD contributed to analysis, critical revision, and final approval of the manuscript. KW, SL and DV contributed to data collection, critical revision, and final approval of the manuscript. JW contributed to design, interpretation of the data, critical revision, and final approval of the manuscript. LS contributed to conception and design, critical revision, and final approval of the manuscript. MW contributed to conception and design, data collection, interpretation of the data, financial support, analysis, manuscript writing, and final approval of the manuscript. All authors read and approved the final manuscript.

\section{Acknowledgements}

This trial is partially funded by a research grant from COOK Medical Inc. (Bloomington, IN, USA).

\section{Author details}

${ }^{1}$ Department of Surgery, Division of Vascular- and Transplant Surgery, Radboud University Nijmegen Medical Centre, Geert Grooteplein-Zuid 10, Nijmegen, GA 6525, the Netherlands. 'Department of Nephrology, Nijmegen, the Netherlands. ${ }^{3}$ Department of Epidemiology Biostatistics and HTA, Nijmegen, the Netherlands. ${ }^{4}$ Department of Radiology, Radboud University Nijmegen Medical Centre, Nijmegen, the Netherlands. ${ }^{5}$ Department of Surgery, Division of Vascular Surgery, Slingeland Hospital, Doetinchem, the Netherlands.

\section{Received: 10 September 2013 Accepted: 1 April 2014} Published: 11 April 2014

\section{References}

1. Nash K, Hafeez A, Hou S: Hospital-acquired renal insufficiency. Am J Kidney Dis 2002, 39:930-936.

2. Hou SH, Bushinsky DA, Wish JB, Cohen JJ, Harrington JT: Hospital-acquired renal insufficiency: a prospective study. Am J Med 1983, 74:243-248.

3. McCullough PA, Wolyn R, Rocher LL, Levin RN, O'Neill WW: Acute renal failure after coronary intervention: incidence, risk factors, and relationship to mortality. Am J Med 1997, 103:368-375.

4. McCullough PA: Contrast-induced acute kidney injury. J Am Coll Cardiol 2008, 51:1419-1428.

5. Mitchell AM, Jones AE, Tumlin JA, Kline JA: Incidence of contrast-induced nephropathy after contrast-enhanced computed tomography in the outpatient setting. Clin J Am Soc Nephrol 2010, 5:4-9.

6. Morcos SK, Thomsen HS, Webb JA: Contrast-media-induced nephrotoxicity: a consensus report. Contrast Media Safety Committee, European Society of Urogenital Radiology (ESUR). Eur Radiol 1999, 9:1602-1613.

7. Solomon RJ, Mehran R, Natarajan MK, Doucet S, Katholi RE, Staniloae CS, Sharma SK, Labinaz M, Gelormini JL, Barrett BJ: Contrast-induced nephropathy and long-term adverse events: cause and effect? Clin J Am Soc Nephrol 2009, 4:1162-1169.

8. From AM, Bartholmai BJ, Williams AW, Cha SS, McDonald FS: Mortality associated with nephropathy after radiographic contrast exposure. Mayo Clin Proc 2008, 83:1095-1100.

9. Rudnick MR, Goldfarb S, Wexler L, Ludbrook PA, Murphy MJ, Halpern EF, Hill JA, Winniford M, Cohen MB, VanFossen DB: Nephrotoxicity of ionic and nonionic contrast media in 1196 patients: a randomized trial. The lohexol Cooperative Study. Kidney Int 1995, 47:254-261.

10. Levy EM, Viscoli CM, Horwitz RI: The effect of acute renal failure on mortality. A cohort analysis. JAMA 1996, 275:1489-1494.
11. Rihal CS, Textor SC, Grill DE, Berger PB, Ting HH, Best PJ, Singh M, Bell MR, Barsness GW, Mathew V, Garratt KN, Holmes DR Jr: Incidence and prognostic importance of acute renal failure after percutaneous coronary intervention. Circulation 2002, 105:2259-2264.

12. European Society of Urogenital Radiology: ESUR guidelines on contrast media. Vienna: ESUR; 2012.

13. American College of Radiologists: Practice guideline for the use of intravascular contrast media. Reston, VA: ACR; 2013.

14. CBO guideline: Precautions iodinated contrast-media. Dutch: CBO guideline; 2007.

15. McCullough PA, Adam A, Becker CR, Davidson C, Lameire N, Stacul F, Tumlin J, Panel CINCW: Risk prediction of contrast-induced nephropathy. Am J Cardiol 2006, 98:27K-36K.

16. Trivedi HS, Moore H, Nasr S, Aggarwal K, Agrawal A, Goel P, Hewett J: A randomized prospective trial to assess the role of saline hydration on the development of contrast nephrotoxicity. Nephron Clin Pract 2003, 93:C29-C34.

17. Balemans $C E$, Reichert $L$, Van Schelven BI, van den Brand JA, Wetzels JF: Epidemiology of contrast material-induced nephropathy in the era of hydration. Radiology 2012, 263:706-713.

18. Kim SM, Cha RH, Lee JP, Kim DK, Oh KH, Joo KW, Lim CS, Kim S, Kim YS: Incidence and outcomes of contrast-induced nephropathy after computed tomography in patients with CKD: a quality improvement report. Am J Kidney Dis 2010, 55:1018-1025.

19. Brar SS, Shen AY, Jorgensen MB, Kotlewski A, Aharonian VJ, Desai N, Ree M, Shah Al, Burchette RJ: Sodium bicarbonate vs sodium chloride for the prevention of contrast medium-induced nephropathy in patients undergoing coronary angiography: a randomized trial. JAMA 2008, 300:1038-1046.

20. Vasheghani-Farahani A, Sadigh G, Kassaian SE, Khatami SM, Fotouhi A, Razavi SA, Mansournia MA, Yamini-Sharif A, Amirzadegan A, Salarifar M, Sadeghian S, Davoodi G, Borumand MA, Esfehani FA, Darabian S: Sodium bicarbonate plus isotonic saline versus saline for prevention of contrast-induced nephropathy in patients undergoing coronary angiography: a randomized controlled trial. Am J Kidney Dis 2009, 54:610-618

21. Sendeski MM: Pathophysiology of renal tissue damage by iodinated contrast media. Clin Exp Pharmacol Physiol 2011, 38:292-299.

22. Evans RG, Ince C, Joles JA, Smith DW, May CN, O'Connor PM, Gardiner BS: Haemodynamic influences on kidney oxygenation: clinical implications of integrative physiology. Clin Exp Pharmacol Physiol 2013, 40:106-122.

23. Murry $C E$, Jennings $R B$, Reimer KA: Preconditioning with ischemia: a delay of lethal cell injury in ischemic myocardium. Circulation 1986, 74:1124-1136.

24. Przyklenk K, Bauer B, Ovize M, Kloner RA, Whittaker P: Regional ischemic 'preconditioning' protects remote virgin myocardium from subsequent sustained coronary occlusion. Circulation 1993, 87:893-899.

25. Wever KE, Warle MC, Wagener FA, van der Hoorn JW, Masereeuw R, van der Vliet JA, Rongen GA: Remote ischaemic preconditioning by brief hind limb ischaemia protects against renal ischaemia-reperfusion injury: the role of adenosine. Nephrol Dial Transplant 2011, 26:3108-3117.

26. Kanoria S, Jalan R, Seifalian AM, Williams R, Davidson BR: Protocols and mechanisms for remote ischemic preconditioning: a novel method for reducing ischemia reperfusion injury. Transplantation 2007, 84:445-458.

27. Whittaker P, Przyklenk K: Remote-conditioning ischemia provides a potential approach to mitigate contrast medium-induced reduction in kidney function: a retrospective observational cohort study. Cardiology 2011, 119:145-150.

28. Er F, Nia AM, Dopp H, Hellmich M, Dahlem KM, Caglayan E, Kubacki T, Benzing T, Erdmann E, Burst V, Gassanov N: Ischemic preconditioning for prevention of contrast medium-induced nephropathy: randomized pilot RenPro Trial (Renal Protection Trial). Circulation 2012, 126:296-303.

29. Manske CL, Sprafka JM, Strony JT, Wang Y: Contrast nephropathy in azotemic diabetic patients undergoing coronary angiography. Am J Med 1990, 89:615-620.

30. Vogl TJ, Honold E, Wolf M, Mohajeri H, Hammerstingl R: Safety of iobitridol in the general population and at-risk patients. Eur Radiol 2006, 16:1288-1297.

31. Loewe C, Becker CR, Berletti R, Cametti CA, Caudron J, Coudyzer W, De Mey J, Favat M, Heautot JF, Heye S, Hittinger M, Larralde A, Lestrat JP, Marangoni R, Nieboer K, Reimer P, Schwarz M, Schernthaner M, Lammer J: 64-Slice CT angiography of the abdominal aorta and abdominal arteries: 
comparison of the diagnostic efficacy of iobitridol $350 \mathrm{mgl} / \mathrm{ml}$ versus iomeprol $400 \mathrm{mgl} / \mathrm{ml}$ in a prospective, randomised, double-blind multi-centre trial. Eur Radiol 2010, 20:572-583.

32. $L i L, L i G, Y U C, L i Y$ : The role of remote ischemic preconditioning on postoperative kidney injury in patients undergoing cardiac and vascular interventions: a meta-analysis. J Cardiothorac Surg 2013, 8:43.

33. D'Ascenzo F, Cavallero E, Moretti C, Omede P, Sciuto F, Rahman IA, Bonser RS, Yunseok J, Wagner R, Freiberger T, Kunst G, Marber MS, Thielmann M, Ji B, Amr YM, Modena MG, Zoccai GB, Sheiban I, Gaita F: Remote ischaemic preconditioning in coronary artery bypass surgery: a meta-analysis. Heart 2012, 98:1267-1271.

34. Mehta Oza N, Raman SV: Letter by Mehta Oza and Raman regarding article, "Ischemic preconditioning for prevention of contrast medium-induced nephropathy: randomized pilot RenPro Trial (Renal Protection Trial)". Circulation 2013, 127:e535.

35. Harjai KJ, Raizada A, Shenoy C, Sattur S, Orshaw P, Yaeger K, Boura J, Aboufares A, Sporn D, Stapleton D: A comparison of contemporary definitions of contrast nephropathy in patients undergoing percutaneous coronary intervention and a proposal for a novel nephropathy grading system. Am J Cardiol 2008, 101:812-819.

doi:10.1186/1745-6215-15-119

Cite this article as: Sterenborg et al:: Remote ischemic preconditioning to reduce contrast-induced nephropathy: study protocol for a randomized controlled trial. Trials 2014 15:119.

\section{Submit your next manuscript to BioMed Central and take full advantage of:}

- Convenient online submission

- Thorough peer review

- No space constraints or color figure charges

- Immediate publication on acceptance

- Inclusion in PubMed, CAS, Scopus and Google Scholar

- Research which is freely available for redistribution 